$$
\text { "tmcs-korandi" — 2012/12/3 - 11:50 — page 375 — \#1 }
$$

\title{
Mathematics in Good Will Hunting I: The mathematicians in Good Will Hunting
}

\author{
József Korándi and Gabriella Pluhár
}

Abstract. This is the first part of a three paper long series exploring the role of mathematicians and of the mathematical content occurring in popular media. In particular, we analyze the movie Good Will Hunting. In the present paper we investigate stereotypes about mathematicians living in the society and appearing in Good Will Hunting.

Key words and phrases: mathematics in media, Good Will Hunting, popularize mathematics.

ZDM Subject Classification: U84, U85, U89, A44, A45, A49.

\section{Introduction}

The image existing in our society about mathematics is important not only for the development and applicability of mathematics but for future mathematicians, as well. We can differentiate three parts of the society based on their relation to mathematics. They play different roles in our society and their evolution has been affected by the division of labour. It is important to understand what is their relation to mathematics, what their purpose is and how they want to achieve it. The three groups are the following:

(1) Sci: the mathematicians' community (representing the image of mathematics based on paradigm system of science),

The work/publication is supported by the TÁMOP-4.2.2/B-10/1-2010-0024 project. The project is co-financed by the European Union and the European Social Fund.

Copyright (C) 2012 by University of Debrecen 


$$
\text { "tmcs-korandi" — 2012/12/3 - 11:50 — page 376 — \#2 }
$$

(2) Edu: Educators' community (including both the people in education and the education institutes),

(3) Pers: the people (representing the individuals' image of mathematics, which may include some mathematical knowledge and competency, which does not necessarily exist but is expected by the society).

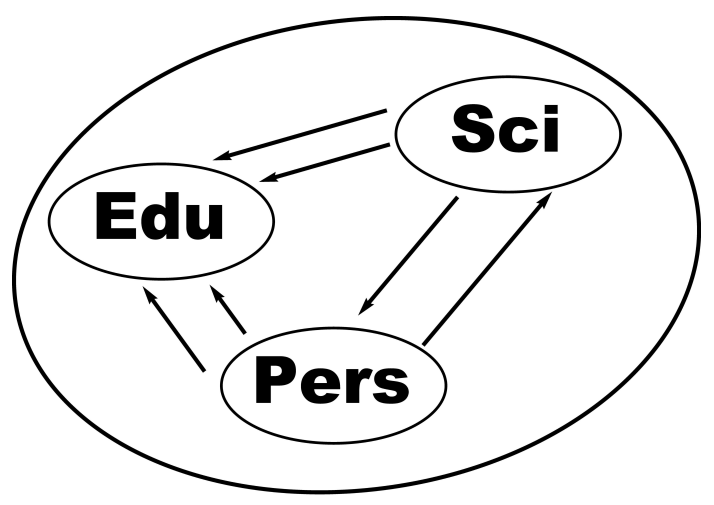

Figure 1. Three groups of society

These groups interact with each other. The relation between Sci and Edu manifests mostly via teacher training. The connection between Edu and Pers is obvious: on the one hand this is the mathematical education, on the other hand it is the society's feedback on what should be taught by the "educators". The first effect is direct, while the other is usually indirect. The interaction between Sci and Pers is quite hidden. Here, we are going to examine this latter interaction, in particular how the appearance of mathematicians and mathematical content in the media affects the individuals.

Mathematicians appear in two very different ways in the media:

- Via scientific documentaries.

This is actually an alternative way of teaching mathematics. It differs from the public education essentially: it teaches through the different media and it needs to capture the individual's attention competing with other members of the show business, while public education is "forced" upon the individual by the government.

- Via popular culture. 
"tmcs-korandi" — 2012/12/3 - 11:50 — page 377 — \#3

The effect pop-culture has on individuals should not be underestimated. Most members of the society, especially when they are young, do not meet mathematicians. Thus their image of a mathematician is shaped by the mathematician figures and their activities appearing in the media. Then they make their carrier choices accordingly. Moreover, the media (mostly movies and television shows) can significantly influence one's interest towards a particular topic. The viewer emotionally connects to the mathematician characters and to the mathematical content. If this emotion is a positive one, it will have a more lasting effect than an actual knowledge learnt on a regular class. This of course does not substitute the impact of mathematics instruction at school-time, but this way teachers may reach out to students who otherwise would lose interest in learning "regular" mathematics.

Fortunately, the creators of a show do not need to compromise on how they portray mathematicians or mathematical content. Proper portrayal is necessary (or rather would be necessary), as recently the people seem to have an image of mathematicians as to be respected, but not aspired to. Even to the extent, that a perfectly average person showing up as a mathematician would already have a positive effect. Such a negative judgment of mathematicians can lead to very twisted real life situations. For example, one of our students in our "Mathematics in Media" course admitted that he keeps his mathematician identity as secret when socializing with people he has not met before. He introduces himself as a law student, rather than a mathematician student.

The class called "Mathematics in Media" was introduced six years ago in 2006 for the BSc Mathematics students at Eötvös Loránd University of Arts and Sciences (ELTE). The idea of the subject was about how to popularize and transmit mathematics to people outside the mathematical community. Probably, there is no need to explain to the reader how important it is to accentuate the humanness and accessibility of mathematics to a general audience. Among other topics, the class involved thorough examination of mathematics and mathematicians in major movies and TV-shows. The first author, who has run the class, observed that the mathematical context and mathematical background did not touch even the best mathematics students. They just accepted that some mathematics is done, and followed the streamline of the story. They claimed that the emotional effect of the movie put a bigger impression on them than the mathematics, and the mathematical content had not been showed long enough to contemplate on it. Nevertheless, as we shall see, the mathematical context can be complex and interesting at the same time, hence a good example of popularizing mathematics
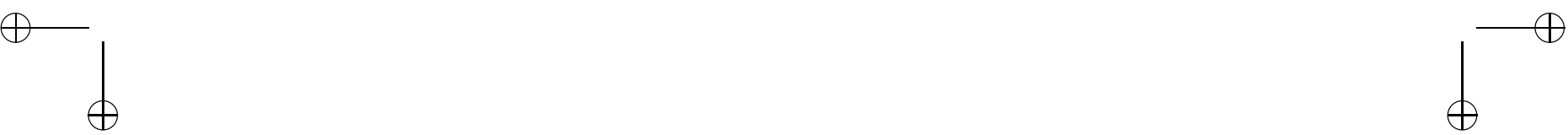


$$
\text { "tmcs-korandi" — 2012/12/3 - 11:50 — page 378 — \#4 }
$$

to students on several levels. It is not a surprise that already videos of lectures are around on the web (e.g. [13]).

This is a 3 paper long series investigating the role of popular culture by analyzing it through an actual example, through the movie titled "Good Will Hunting". This is the first paper, where we study the image of mathematicians projected by the movie. In the second paper [5] we explore the actual mathematical content from the mathematicians' and the mathematics teachers' perspective. Finally, the third paper [6] considers how the mathematical content can be integrated into high school education.

\section{Preliminaries}

Several popular beliefs (called stereotypes) exist about every profession in the society. Whenever such a belief is repulsive, children are less likely to orient towards the particular profession. How appealing a profession is changes over time. Nowadays, for example, being a "media man" is much more popular than being a mathematician.

The stereotypes far from reality can have a harmful impact. If one chooses a job portrayed in a much better way than it is in reality, then they might only realize after several years that this job does not fit them. Or, on the other hand, if a profession is surrounded by a negative public opinion (like the mathematicians'), people may turn away form it even if they could be successful in it. Moreover, the students learn mathematics less enthusiastically because they think (influenced by the society's view) that to be successful in mathematics one has to be a very special person which they would never be.

However, the parents themselves play an important role on how their children choose professions. Many children follow the steps of their parents (e.g. artists' children become artists themselves, etc.) not only because of genetics, but rather because the parents set an appealing example and bring the profession close to their children. The effect of the media on children and of their parents is very important in the evolution of different stereotypes. As most of the people do not know mathematicians closely, their image on a mathematician is solely based on what they see in the media. This affects both the social acceptance of mathematicians and whether the young would choose to be a mathematician as their profession. According to [11], 45.3\% of elementary school students of age 11 consider the media as their basic input on their idea about scientists. It would be natural to think that people base their image of mathematicians on 


$$
\text { "tmcs-korandi" — 2012/12/3 - 11:50 — page 379 — \#5 }
$$

their mathematics teachers. Nevertheless, according to Berry-Picker's research [1], the students do not consider their mathematics teacher to be mathematician.

In this paper we examine the common opinion about mathematicians, mainly how they are portrayed in the media and in the particular movie Good Will Hunting. This is going to be shown by observing the following aspects of mathematicians in the movie:

- appearance (physical abilities, clothing, environment),

- social place,

- emotional life,

- relationships (social relationships, relations at work, private life),

- mentality (at work, at other parts of life),

- methods of work.

\section{Popular beliefs about scientists}

Stereotypes about scientists exist in society for centuries. People can access them by looking at old paintings and reading literary works. Scientific research investigating these beliefs started in the middle of 1900s and are popular nowadays, as well.

In 1957 Mead and Metraux [7] published a paper based on the essays of 35.000 high school students. The students had to write about what kind of image they have about scientists. The research showed that according to the students the typical scientist is male, old or middle aged, wears glasses and a white gown, usually has a beard, works in a laboratory, deals with dangerous and mysterious objects secretly, and is mysterious, as well. The scientists carefully write notes into their black note-books, sometimes shouting enthusiastically, like e.g. "I have found it!". They discover things which can be used to create better products, and they read books all the time.

Chambers [2] published the results of his researches in 1983. He developed a method which is called DAST (Draw-A-Scientist Test) by the literature. More than 4800 children were asked to draw a picture of a scientist. By analyzing the drawings it turned out that many properties are connected with scientists in the children's thoughts already at a very early age. These features were the following: laboratory gown, glasses, facial hair, the symbols of research such as laboratory furniture, scientific equipments, the symbols of knowledge like books, bookshelves, the products of science and formulas etc. Only girls drew female 


$$
\text { "tmcs-korandi" — 2012/12/3 - 11:50 — page 380 — \#6 }
$$

scientists, but hardly more than $1 \%$ of them. This is surprising because if someone is simply asked to draw a person, then in most of the cases they draw someone of the same gender. On the pictures the scientist always works in a room or in a laboratory, usually in the basement. On some of the drawings the scientist works on mysterious or dangerous things. Furthermore, on many drawings the fictional scientist-figures appeared as a Frankenstein or as a Jekyll-Hyde type figure.

Schebeci and Sorensen [10] investigated how much the image of scientists differ for different ethnic groups. They found that the images do not differ, and claimed this to be an effect of the media: e.g. very similar movies can be seen all over the world. This observation was also found by Finson [3].

It should be mentioned that stereotypes about scientists has been researched by others, as well. For example, Odell, Hewitt, Bowman and Boone [8] improved the DAST method, they questioned not only primary and secondary school students, but even students from universities. Rampal in [9] questioned starting teachers to comment on their views of scientists. Several new properties have been found among the answers. According to them, scientists are emotionless, careless and lack social sensibility. Thus the stereotypes slowly change over time. It seems, for example, that the crazy scientist view or the mysterious factor are starting to disappear. Many researchers think that the media (more precisely television, movies and series) is solely responsible for this effect.

To finish the historical overview we present some research results on stereotypes particularly about mathematicians. Berry and Picker [1] asked 13 years old students in Plymouth (United Kingdom) and in New York (USA) to draw a mathematician. Then the researchers talked to the children about their drawings. The image of a mathematician turned out to be very similar to the image of a scientist. It seems that the social view is almost the same about them. By the drawings and discussion the mathematician is a man, does not have friends except other mathematicians, he is lonely, white, usually fat, old-fashioned and wears glasses. The mathematician's forehead is wrinkled from the lots of thinking, gets angry very fast, he is bald or has bizarre hair. There are pencils, pens, calculators all around him, and he writes inexplicable formulas on a board. Furthermore, it turned out that the students hardly know anything or have any idea about what a mathematician does, or how mathematics can be useful. Some of the answers to this particular question were the following: the mathematician teaches, works in a bank or a shop, solves difficult problems, hardly ever calculates.

In the research by Wilson and Latterell [12], elementary school students had drawn a picture of a mathematician and then wrote a caption to them. The
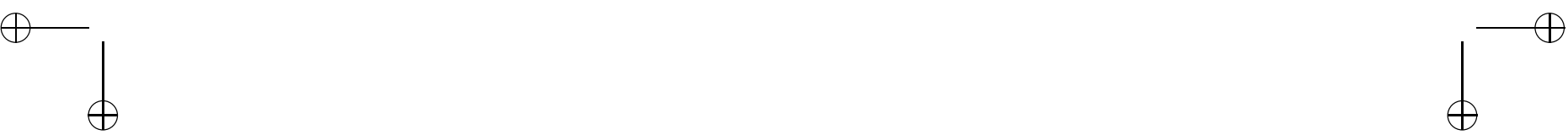


$$
\text { "tmcs-korandi" — 2012/12/3 - 11:50 — page 381 — \#7 }
$$

drawings mostly showed a bald man with glasses and beards, who were working at a blackboard or on a computer. Among the captions these were the most typical ones: mathematicians have no friends, except other mathematicians, they are not married or seeing anyone, they are usually fat and very unstylish, there are wrinkles in their forehead from thinking so hard, they have no social life whatsoever, they are 30 years old and are of a very short temper, mathematicians are people who think really hard and never have sex.

There is a non-representative Norwegian research by Grevholm [4] from 2010. He used drawings and questions to find out what high school students thought about mathematicians and about mathematics. Here the following features were dominant: The mathematician is bald or has extraordinary hair, usually wears glasses, is surrounded by formulas, works alone, is lonely. The mathematicians seem to be happy only on a few pictures, and the children think that their work is connected to numbers and calculations.

\section{Scientists in the media}

Wilson and Latterell [12] lists many literary works and movies featuring mathematicians, as well. In these the mathematician is mostly puzzled, at some places even mad. This partially comes from the stereotypes and partially from the fact that in the movies the main character's spiritual nature or illness forms the basis of the conflict. The director chooses such a main character to build the dramatic action on this situation. Although, the creator choosing a mathematician as the main character is an indicator of the existing stereotypes.

The scientist's image changes over time. This can be observed from how the different movies portray scientist characters. This image is more or less in accordance to the one existing in the society. Most of the attributes are exactly the same and do not change, or only very slowly. Nevertheless, some features change. For example, the image of the mad scientist discovering crazy, terrible things and trying to conquer the world is slowly disappearing from both the screen and from the research results. While in three out of the seven James Bond movies created in the $60 \mathrm{~s}$ featured a genius, but mad scientist, since then no enemy of the 007 agent has been such a character. Another example of a literary work supporting an unconventional scientist image is the popular television series Numb3rs. Here, one of the main characters is a young mathematician who is handsome, thin, clever, does not wear glasses, has lots of friends, has a girlfriend, goes out often, 


$$
\text { "tmcs-korandi" — 2012/12/3 - 11:50 — page } 382 \text { — } 8
$$

and has a social life. This shows a unique mathematician figure living a normal life, which very much differs from the previously presented ones.

We are going to take a closer look at Good Will Hunting. Good Will Hunting is a 1997 American drama film directed by Gus Van Sant. It won several awards, e.g. Ben Affleck and Matt Damon received an Academy Award for the screenplay and Robin Williams received an Academy Award for forming the psychologist. Good Will Hunting is not primarily about mathematics and not even about the life of a mathematician. Nevertheless, mathematicians and mathematical content are present. The main actor, Will Hunting (Matt Damon) is a twenty year old person from South Boston, but he doesn't attend university classes. He is a janitor at the Massachusetts Institute of Technology (MIT). He lives in a desolate suburban area, and because of his temper he often crosses paths with the law. However, he stands out of this depraved environment with his extraordinary abilities. He has the intellectual skills of a genius, but he cannot evolve them due to his social background. At least not for a while. The movie shows how he turns his life into a more successful one with the help of a psychologist coming from a similar social background. The story is basically an intellectual Rocky-story, the main difference being that the main character builds a carrier in science instead of in sports. It is not surprising that the script received an Academy Award. The aim of the present paper is not to analyze the movie, but rather to examine the mathematician image it portrays. We examine the mathematical content in [5] and $[6]$.

\section{Good Will Hunting and the stereotypes}

First we explore what stereotypes on mathematicians are present in this film. That is, we examine the characters from the following perspectives (in brackets we listed the stereotype): gender (male), age (old), race (white), build (fat), facial hair (present), glasses (present), clothing (old-fashioned), hair (bald or bizarre hair), relationships (no friends, lonely) and work (surrounded by formulas, thinking about mysterious things). There are eight characters in the movie connected to mathematics in some way. They are Will Hunting, professor Gerald Lambeau, Tom, Alexander, Steven, Jack, Nimesh and "Alison" (she is the only female character in the movie connected to mathematics, we refer to her using the actor's first name). We examined these characters from the listed perspectives and summarized the results in Tables 1 and 2 . 
Table 1. Summary of Will's, Lambeau's, Tom's and Alexander's look by the given aspects (* Reading glasses)

\begin{tabular}{|l||l|l|l|l|}
\hline & Will & Lambeau & Tom & Alexander \\
\hline \hline age & young & middleaged & young & elderly \\
\hline gender & male & male & male & male \\
\hline race & white & white & white & white \\
\hline build & slim & slim & slim & slim \\
\hline facial hair & no & no & no & no \\
\hline glasses & no & no* $^{*}$ & yes & no* \\
\hline clothing & casual & casual & formal & formal \\
\hline hair & usual & usual & usual & balding \\
\hline
\end{tabular}

Table 2. Summary of Steven's, Jack's, Nimesh's and "Alison's" look by the given aspects

\begin{tabular}{|l||l|l|l|l|}
\hline & Steven & Jack & Nimesh & "Alison" \\
\hline \hline age & young & young & young & young \\
\hline gender & male & male & male & female \\
\hline race & white & black & hindu & white \\
\hline build & slim & slim & slim & overweight \\
\hline facial hair & no & moustache & Van Dyke beard & no \\
\hline glasses & no & no & no & no \\
\hline clothing & casual & casual & casual & old-fashioned \\
\hline hair & usual & balding & long & unsuitable \\
\hline
\end{tabular}

Good Will Hunting supports the white male stereotype of mathematicians. Seven of the eight characters are male, five of them is white: Will, Lambeau, Tom, Alexander and Steven. Jack is black, Nimesh is hindu, and the only female mathematics student is white, as well. The movie does not fit the stereotype concerning the age: Alexander, being in his fifties, is the oldest, and the five mathematics students are fairly young. None of the characters are fat. "Alison" looks to be slightly overweight, but this might as well be a result of her oldfashioned dressing style. Two of the male characters have some facial hair and only one of them wears glasses (though Alexander and Lambeau uses reading glasses). The characters usually dress in a non-formal way, Lambeau even teaches having a scarf casually thrown around his neck. Their hair is quite usual, there are nothing bizarre in them, two of the eight characters are balding. 


$$
\text { "tmcs-korandi" — 2012/12/3 - 11:50 — page 384 — \#10 }
$$

Maybe Alexander has the most of the usual stereotypes about mathematicians: he is a middle-aged, balding white male, has wrinkles on his forehead. Although he uses his glasses only for reading, the viewer may associate him wearing glasses as he does that one third of the time he can be seen on the screen. Tom is another type of the "typical" mathematician: the smart but not genius, hardworking, reliable and boring mathematician. Finally, let us mention that "Alison" is very fitting for a female mathematician: she dresses in an old-fashioned way, her hair is not really appealing, and her whole appearance lacks of the feminine touch one expects from most women.

Concerning friends, family and social relationships there is no uniform picture in the movie. Professor Lambeau seems to be a man of the world, he is as successful in making relationships as in his work. Despite of the fact that Will has a wide, strong circle of friends and even has a girlfriend he is emotionally damaged and socially deviant. And here the stereotype hits again: Will is the genius.

A typical element of the stereotype about mathematicians is that they are not only clever, but very clever. This is used by the makers of Good Will Hunting: it turns out that Will is amazingly talented in many different areas. For example, when he goes to the Harvard bar with his friends, Will humiliates a history student by knowing more history without education than him. Later, when he is tried by a court for fighting, it turns out that he is familiar with most of the criminal cases in the United States of America (USA). This is a most valuable skill, as in the USA the legal system is based on precedent cases. Therefore, the audience can see that Will Hunting could be a great lawyer or a historian, as well. Nevertheless, the creators decided to pick mathematics to illustrate Will's extraordinary talent. He is a genius in mathematics, he is even better than the professor who won the Fields Medal.

\section{Doing mathematics in the movie}

What do mathematicians do when they work? Most of the people do not have any idea about it, and Good Will Hunting does not necessarily help them have a proper view about it. Right after the opening credits, mathematics is presented in the film. A big lecture hall of MIT and the last minutes of a mathematics lecture can be seen. There are four huge blackboards full with formulas. This supports the public opinion that mathematicians work with formulas. Professor Lambeau announces to the students that there is a Fourier transformation written on the 


$$
\text { "tmcs-korandi" — 2012/12/3 - 11:50 — page 385 — \#11 }
$$

notice-board of the department. If one can solve it until the end of the semester, then its solution will be published in the journal of the university (MIT Tech). Then the professor names several people who already published in MIT Tech. This scene backs up the stereotype that mathematicians solve problems and they think a lot to solve a single exercise. Nevertheless, the particular exercise on the board has nothing to do with Fourier transformations (in [5] we explain the background in detail), and is not particularly hard, either. There are two things we should mention here which are specific to the Hungarian dubbed version of Good Will Hunting. According to the Hungarian translation this exercise has been solved and the solution published by many people over and over again. This obviously false idea shows the Hungarian society's ignorance on mathematician's work. Even the name Fields in one of the most respected awards was translated into Hungarian ("pálya").

In the next scene containing any mathematics is the one where Will writes something on his mirror with a black marker. He solves the professor's problem right away, he writes the answers without even thinking about them. This scene sends the message that if someone is a genius, then they do not have to do any calculations. He solves even the very difficult problems immediately, which would take a semester for other people to solve. This, of course, gives a false view about good mathematicians. There are calculations which need to be written down, even for the most brilliant mathematicians.

The film featuring mathematicians working in pairs or groups correlates with reality. Professor Lambeau even talks about the work of the whole department when he announces the problems. Later he solves a graph coloring problem together with Will. In another scene Lambeau, Tom and Alexander work together, then Will joins them. These scenes contradict the stereotype about lonely mathematicians. Nevertheless, the movie show that mathematicians can and do work alone, as well, not only in teams.

The mathematical terminology is continuously present in the movie, probably even more so than would be necessary. The film starts off with some formulas swirling in the background very mysteriously during the opening credits. The boards would naturally be full of formulas, but the characters use jargon even when it would not be essential. For example, the second problem on the departmental notice board is about homeomorphically irreducible graphs. It sounds very "scientific" and difficult. Nevertheless, a homeomorphically irreducible graph is simply a graph without points with degree 2, i.e. without points having exactly 2 edges. This and similar scenes filled with mathematical terminology suggest 


$$
\text { "tmcs-korandi" — 2012/12/3 — 11:50 — page 386 — \#12 }
$$

to the viewer that mathematics is not understandable by ordinary people. This phenomenon can make people admire mathematics to a certain extent, but it ultimately distances the audience from mathematics, makes some people afraid of it. We believe that in Hungary the situation is slightly better than shown in the movie. For example, after asking several graph theorists, we found that there is no particular Hungarian terminology used for homeomorphically irreducible trees.

In the movie the joy of doing mathematics is not present at all. There are scenes where the mathematicians talk to each other very passionately when solving a problem. Will, the genius of the century, is quite emotionless: he solves the difficult problems with some interest, but he claims to be quite bored since the problems do not challenge him. However, no mathematician or student appear who works on a problem with joy or excitement. Furthermore, nobody seems to admire a beautiful solution of a given problem or exercise. It seems from the movie that doing mathematics is a very tiring and boring activity. Therefore, the mathematician characters are not attractive figures, and nobody would want to be like them.

In our Mathematics in Media class we have questioned university students about Good Will Hunting. For some of them it was really inspiring that it is possible to do mathematics effortlessly and solve mathematical problems even at a young age. This was suggested to them by the character of Will Hunting. However, they all consider Will a fictional character, and they do not believe that someone with similar skills could exist in the real world.

Thus, it can be said that the image of mathematicians and mathematics given by Good Will Hunting is not attractive at all. The audience sees mathematics to be a very complicated thing. Most of it is difficult even for mathematicians. They suffer several failures, and they are only able to solve problems with a tiring work. Furthermore, even if a professor achieves some honor at the top of his carrier, like Lambeau or Alexander in the movie, the possibility still exists that a bright young boy can make all of their work of life meaningless. The movie suggests to the audience that mathematics is a mysterious, horrible thing, and being a mathematician is a very unpleasant way to live a life.

\section{Conclusion}

In this paper we examined what kind of stereotypes about mathematicians appear in the movie titled Good Will Hunting. It turned out that the movie supports most of the popular beliefs, but it contradicts others in some points. There 


$$
\text { "tmcs-korandi" — 2012/12/3 — 11:50 — page 387 — \#13 }
$$

exists a lot of research on social beliefs about scientists and mathematicians. It seems that the popular image is negative, does not depend on the nationality or ethnicity of the people. These phenomenons are attributed to the media. Furthermore, the media has an important role in keeping, strengthening or weakening these stereotypes. It seems probable that keeping the status quo is mostly due to the fact that the media features mathematicians supporting the popular image, which in turn affects this image. The media (by showing a more popular and/or more realistic picture about scientists) could improve the public opinion about unpopular professions and could make their life and work look more tempting for both children and parents.

\section{References}

[1] J. Berry and S. H. Picker, Your pupils' images of mathematicians and mathematics, Mathematics in School (Harlow) 29, no. 2 (2000), 24-26.

[2] D. W. Chambers, Stereotypic images of the scientist: The Draw-a-Scientist Test, Science Education 67, no. 2 (1983), 255-265.

[3] K. D. Finson, Applicability of the DAST-C to the images of scientists drawn by students of different racial groups, 2001, Paper presented at the annual regional meeting of the North Central Region Association for the Education of Teachers of Science, Madison, WI.

[4] B. Grevholm, Norwegian upper secondary school students' views of mathematics and images of mathematicians, in: Current state of research on mathematical beliefs XVI, (K. Kislenko, ed.), 2010, 120-136, MAVI-16 Conference, June 26-29, 2010, Tallinn, Estonia.

[5] G. Horváth, J. Korándi and Cs. Szabó, Mathematics and Good Will Hunting II, Teach. Math. Comput. Sci. (2013), accepted.

[6] J. Korándi and G. Pluhár, Mathematics and Good Will Hunting III, Teach. Math. Comput. Sci. (2013), submitted.

[7] M. Mead and R. Métraux, Image of the scientist among high-school students: A pilot study, Science 126, no. 3270 (Aug. 1957), 384-390.

[8] M. R. I. Odell, P. Hewitt, J. Bowman and W. J. Boone, Stereotypical images of scientists: A cross-age study, 1993, Paper presented at the 41st annual national meeting of the National Science Teachers Association, Kansas City, MO.

[9] A. Rampal, Images of science and scientists: A study of school teachers' views I. Characteristics of scientists, Science Education 76, no. 4 (1992), 415-436.

[10] R. A. Schibeci and I. Sorensen, Elementary school children's perceptions of scientists, School Science and Mathematics 83, no. 1 (1983), 14-20. 


$$
\text { "tmcs-korandi" — 2012/12/3 — 11:50 — page 388 — \#14 }
$$

[11] H. Türkmen, Turkish primary students' perceptions about scientist and what factors affecting the image of the scientists, Eurasia Journal of Mathematics, Science and Technology Education 4, no. 1 (Feb. 2008), 55-61.

[12] J. L. Wilson and C. M. Latterell, Nerds? Or nuts? Pop culture portrayals of mathematicians, ETC: A Review of General Semantics 58, no. 2 (2001), 172-178.

[13] R. Wilson, A millenium of mathematical puzzles: Homeomorphically irreducible trees, http://www . youtube. $\operatorname{com} /$ watch?v=811LbompjPg.

JÓZSEF KORÁNDI

MATHEMATICS TEACHING AND EDUCATION CENTRE

EÖTVÖS LORÁND UNIVERSITY

1117 BUDAPEST, PÁZMÁNY PÉTER SÉTÁNY 1/C

HUNGARY

E-mail: korandi@cs.elte.hu

GABRIELLA PLUHÁR

DEPARTMENT OF ALGEBRA AND NUMBER THEORY

EÖTVÖS LORÁND UNIVERSITY

1117 BUDAPEST, PÁZMÁNY PÉTER SÉTÁNY 1/C

HUNGARY

E-mail: plugab@cs.elte.hu

(Received February, 2012) 